\section{Was ist der Vorteil?}

\section{Die hier vorgestellte Studie vergleicht die Patientenspezifische Instrumentierung (PSI) der Knie-TEP mit computerassistierter und konventioneller Technik. Postoperativ zeigte sich ein ver- gleichbares Prothesenalignment im Vergleich zur computer- assistierten Technik und ein besseres Alignment im Vergleich zur konventionellen Technik. Die Operationszeit der PSI-Grup- pe war am kürzesten. \\ MacDessi SJ et al. A comparison of alignment using patient specific guides, computer navigation and conventional instrumentation in total knee arthroplasty. Knee 2014; 21: 406-409}

\section{Methodik \\ $\nabla$}

In einer retrospektiven Studie wurden 115 Patienten, welche zwischen April 2010 und Februar 2012 eine MRT-basierte patientenspezifische Instrumentierung (PSI) einer Knie-TEP erhielten, mit einer Gruppe von 53 Patienten mit computerassistierter Prothesenimplantation (CAI) und einer Gruppe von 92 Patienten mit konventioneller Prothesenimplantation (KON) verglichen. Postoperativ wurde die Beinachse evaluiert und ein CT zur Beurteilung der Prothesenlage und -rotation angefertigt. Die Ergebnisse werden als prozentualer Anteil der Patienten innerhalb eines als regelrecht definierten Alignments von \pm 3 Grad um die mechanische Beinachse angegeben. Zusätzlich wurde die Operationszeit der jeweiligen Gruppen erhoben.

\section{Ergebnisse}

Postoperativ hatten 91,3\% der PSI-Gruppe ein regelrechtes Alignment. Die CAIGruppe war auf ähnlichem Niveau mit $90,7 \%$, wohingegen die KON-Gruppe einen deutlich niedrigeren Wert von 80,4\% aufwies. Hinsichtlich der Ausrichtung der femoralen Komponente in der coronaren Schichtung hatten 97,4\% der PSI-Gruppe und 96,3\% der CAI-Gruppe ein regelrechtes Alignment. Erneut hatte die KONGruppe einen schlechteren Wert von 89,1\%. Die sagittale Ausrichtung der Femurkomponente zeigte keine statistisch relevanten Unterschiede. Bei der Betrachtung der tibialen Komponente in der coronaren Schichtung hatten 97,4\% der PSIGruppe, $100,0 \%$ der CAI-Gruppe und
88,0\% der KON-Gruppe ein regelrechts Alignment. Es zeigten sich keine signifikanten Unterschiede der Prothesenrotation. Die Operationszeit für die PSI-Gruppe betrug $80,2 \mathrm{~min}$. Sie war signifikant niedriger als die Operationszeit der CAI-Gruppe von 110,2 min und der KON-Gruppe

\section{Kommentar \\ $\nabla$}

Die PSI der Knie-TEP ist derzeit ein vieldiskutiertes Thema. Die hier vorliegende Studie zeigte, dass das mit der PSI erreichte Alignment mit dem der CAI-Gruppe vergleichbar ist. Die KON-Gruppe zeigte schlechtere Werte im Hinblick auf die Beinachse sowie für die coronare Ausrichtung der Femur- und Tibiakomponente. In der sagittalen Ausrichtung und für die Prothesenrotation zeigten sich keine relevon $86,0 \mathrm{~min}$. vanten Unterschiede der PSI- zur KON Gruppe. Jedoch wurde zusätzlich gezeigt, dass mit der PSI die Operationszeit reduziert wurde.

Schwachpunkte der hier vorgestellten Studie sind die fehlende Analyse des funktionellen Ergebnisses und des Kosten/Nutzen-Verhältnisses. Anderl et al. berichteten 2-Jahres-Ergebnisse nach PSI vs. KON. Hier hatte die PSI einen besseren Knee Society Score (Abschnitt Funktion) und ein niedrigeres Schmerzniveau (VAS). Die übrigen Parameter (u.a. Beweglichkeit und WOMAC-Score) zeigten keine wesentlichen Unterschiede [1]. Tibesku et al. führten eine differenzierte Kostenrechnung der PSI im Vergleich zur KON durch. Mehrkosten der präoperativen Bildgebung stehen Einsparungen bei der OP-Zeit und bei OP-Sieben gegenüber. Sie kamen zu dem Schluss, dass die PSI unter bestimmten Voraussetzungen kosteneffektiv ist [2].

\section{Literatur}

1 Anderl W, Pauzenberger L, Kölblinger R et al. Patient-specific instrumentation improved mechanical alignment, while early clinical outcome was comparable to conventional instrumentation in TKA. Knee Surg Sports Traumatol Arthrosc 2014 Oct 19 (epub ahead of print), doi 10.1007/s00167-014$3345-2$

2 Tibesku CO, Hofer P, Portegies W et al. Benefits of using customized instrumentation in total knee arthroplasty: results from an activity-based costing model. Arch Orthop Trauma Surg. 2013, 133:405-411

\section{Dr. med. Leif Claaßen}

Orthopädische Klinik der Medizinischen

Hochschule Hannover

leif.claassen@ddh-gruppe.de

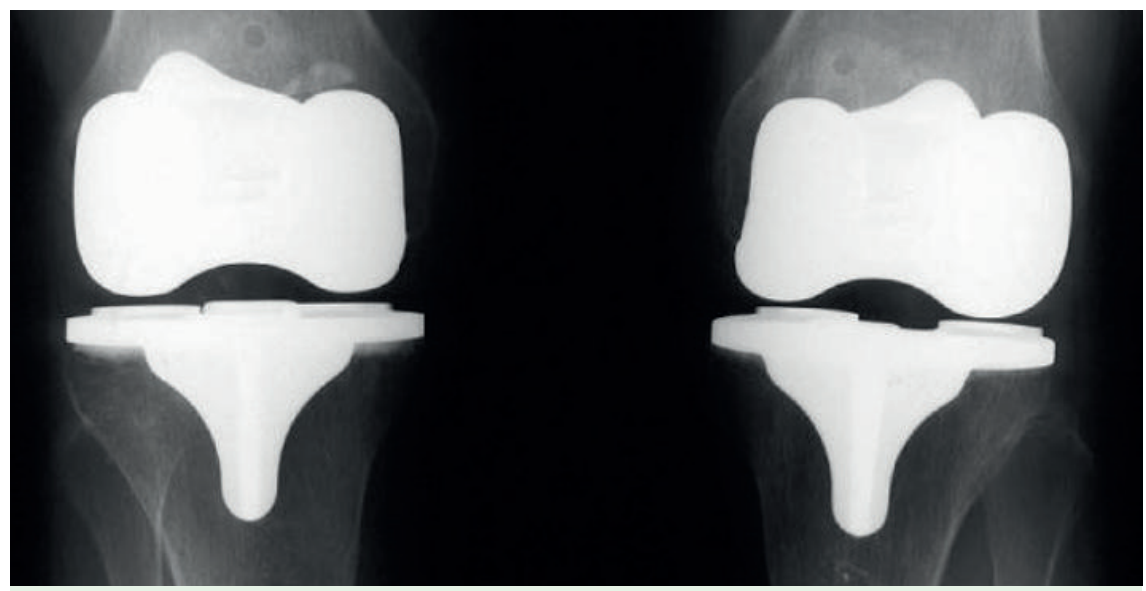

Postoperatives Röntgenbild einer beidseitigen Knie-TEP (Bild: Radmer, S.; Andresen, R.; Sparmann, M. Einzeitiger bilateraler Kniegelenkersatz bei Patienten mit Rheumatoider Arthritis. Z Orthop Unfall 2006; 144: 472 - 476). 